\title{
Time-Frequency Analysis of Heart Rate Variability for Neonatal Seizure Detection
}

\author{
M. B. Malarvili, ${ }^{1}$ Mostefa Mesbah, ${ }^{1}$ and Boualem Boashash ${ }^{1,2}$ \\ ${ }^{1}$ Perinatal Research Centre, School of Medicine, University of Queensland, Herston, QLD 4029, Australia \\ ${ }^{2}$ Signal Processing Research Center, Department of Electrical and Computer Engineering, College of Engineering, \\ University of Sharjah, P.O. Box 27272, Sharjah, United Arab Emirates
}

Received 1 May 2006; Revised 29 January 2007; Accepted 2 February 2007

Recommended by Pablo Laguna Lasaosa

\begin{abstract}
There are a number of automatic techniques available for detecting epileptic seizures using solely electroencephalogram (EEG), which has been the primary diagnosis tool in newborns. The electrocardiogram (ECG) has been much neglected in automatic seizure detection. Changes in heart rate and ECG rhythm were previously linked to seizure in case of adult humans and animals. However, little is known about heart rate variability (HRV) changes in human neonate during seizure. In this paper, we assess the suitability of HRV as a tool for seizure detection in newborns. The features of HRV in the low-frequency band (LF: $0.03-0.07 \mathrm{~Hz}$ ), mid-frequency band (MF: $0.07-0.15 \mathrm{~Hz}$ ), and high-frequency band (HF: $0.15-0.6 \mathrm{~Hz}$ ) have been obtained by means of the timefrequency distribution (TFD). Results of ongoing time-frequency (TF) research are presented. Based on our preliminary results, the first conditional moment of HRV which is the mean/central frequency in the LF band and the variance in the HF band can be used as a good feature to discriminate the newborn seizure from the nonseizure.
\end{abstract}

Copyright (c) 2007 M. B. Malarvili et al. This is an open access article distributed under the Creative Commons Attribution License, which permits unrestricted use, distribution, and reproduction in any medium, provided the original work is properly cited.

\section{INTRODUCTION}

Neonatal epileptic seizures are major indicators of a number of central nervous system (CNS) disorders. A careful assessment of seizures is needed at the early stage to prevent further damages to the brain [1]. Growing attention is focused on the development of computerized methods to automatically detect newborn seizure based on the EEG. There are a number of techniques available for detecting neonatal EEG seizures in the time [2], frequency [3], and time-frequency [4] domains. However, neonatal seizure recognition remains a very challenging task and lacks a reliable detection scheme for clinical use [5]. There is a new tendency towards using information from different physiological signals such as ECG, respiration, and blood pressure to detect seizure [6-9]. This extra information is expected to enhance the performance and robustness of the seizure detectors. This is in line with our longterm goal of using information from different physiological signals such as EEG, ECG, blood pressure, respiration, and oxygen saturation to robustly detect seizures in newborns.

Continuous monitoring of the newborn ECG and heart rate have been successful alternative guides in detecting seizures [10]. In [11], the authors investigated rhythmic changes in ECG and heart rate to alert the physicians to the presence of seizures in 9 paralyzed infants. In addition, the authors in [6] reported that heart rate changes are an extremely common feature of complex partial seizures. Seizures can cause extreme alteration to autonomic activity. ECG and variation in ECG characteristics are primarily under control of the autonomic nervous system (ANS), providing sensitive and noninvasive means of detecting alterations in autonomic activity. Early investigations by neurologists on animal models [7], adults [6-9], and children [12] suggest that paroxysmal changes in ECG, including heart rate, alteration in the RR and QT intervals, are attributed to clinical seizure activity. The conclusions proposed by neurologists are case studies based on the continuous monitoring of the behavior of ECG and EEG channels simultaneously. The precise relationship between these changes and seizures has not been specifically determined.

The HRV is emerging as a major noninvasive tool in monitoring the state of the ANS [13]. The ANS has sympathetic and parasympathetic components. The separate rhythmic contributions from sympathetic and parasympathetic autonomic activities modulate the heart rate, and thus the RR intervals of the QRS complex in the ECG at distinct 
frequencies. Sympathetic activity in newborn is associated with the low-frequency (LF) range $(0.03-0.15 \mathrm{~Hz})$ while parasympathetic activity is associated with the higher-frequency (HF) range $(0.15-0.6 \mathrm{~Hz})$ of the heart rate. The mid-frequency $(\mathrm{MF})$, centered near $0.1 \mathrm{~Hz}$, is both parasympathetically and sympathetically mediated. The HF corresponds to the respiratory and the LF is mediated by a variety of different influences [14].

The HRV characteristics have been investigated with different algorithms based on either time or frequency domains. The main difficulty encountered in frequency-domain processing is the nonstationary behavior of heart beats. Even for a normal healthy person, the heart beats tend to be timevariant. This is because the interbeat interval of the heart rhythm varies markedly due to irregularities in the initiation of the cardiac impulse in the atrium. These nonstationarities become more severe in abnormal cardiac rhythms. TF methods have been introduced to specifically deal with such signals. They are able to provide localized time and frequency descriptions of HRV necessary to characterize such changing autonomic regulation [15].

In this paper, we used the first and second conditional moments of TFD of the HRV in the three frequency bands (LF, MF, and HF) to identify the changes in HRV during seizures. The first conditional moment corresponds to the mean or central frequency of the respective spectrum of interest at a particular time obtained from the TFD while the second conditional moment corresponds to the variance. The purpose of studying these variables is to accurately determine the effect of the seizure on the frequency location of HRV components (LF, MF, and HF) in TF plane. This may in turn allow a clear separation between seizure and nonseizure events.

To realize this, a high-resolution and reduced-interference TFD is needed to clearly separate between the different components in HRV. In [16], it was reported that the TFD conditional moments are able to improve the performance of classification of nonstationary time series compared to those moments based on time or frequency alone.

\section{TIME-FREQUENCY DISTRIBUTIONS}

The Fourier transform (FT) is well suited for the analysis of stationary signals. It gives a representation of the frequency components of the signal but does not allow any localization in time. Since most real-life signals are nonstationary (i.e., their frequency content varies with time), a more global analysis method that represents this type of signals in both time and frequency domain simultaneously is needed.

One of the earliest used time-frequency signal representation is the spectrogram (SP) (defined as the squared magnitude of the short-time Fourier transform (STFT)). The main drawback of the SP is the existence of a tradeoff between time and frequency resolutions. In order to increase the frequency resolution, a long window is required. This choice, however, results in a poor time resolution and also invalidates the assumption of local stationarity. To overcome this limitation, several TFDs have been proposed. One commonly used class
TABLE 1: TFDs and their corresponding kernels.

\begin{tabular}{l|l}
\hline TFDs & Kernel $G(t, \tau)$ \\
\hline SPWVD & $h^{2}(\tau / 2) g(t) ; \quad h(\tau)$ and $g(t)$ are window functions \\
SP & $w(t+\tau / 2) w(t-\tau / 2) ; \quad w(t)$ is an analysis window \\
& function \\
CWD & $\sqrt{\pi \sigma /|\tau| e^{-\pi^{2} \sigma t^{2} / \tau^{2}}}$ \\
MBD & $\cosh ^{-2 \beta}(t) / \int \cosh ^{-2 \beta}(\varsigma) d \varsigma$ \\
\hline
\end{tabular}

of TFDs, of which the spectrogram is a member, is the class of the quadratic shift invariant time-frequency distributions (TFDs) [17]. For a given real-valued signal $x(t)$, these distributions can be parameterized by means of a time-lag kernel $G(t, \tau)$ according to the formula

$$
\rho_{z}(t, f)=\iint G(t-u, \tau) z\left(u+\frac{\tau}{2}\right) \bar{z}\left(u-\frac{\tau}{2}\right) e^{-j 2 \pi f \tau} d u d \tau,
$$

where $\bar{z}$ stands for the complex conjugate of $z$, the analytic associate of $x(t)$ [17]. The time-lag kernel $G(t, \tau)$ determines the characteristics of TFDs and how the signal energy is distributed in the TF plane. Unless otherwise specified, the integration limits are $-\infty$ and $+\infty$. The TFDs used in our investigation are the smoothed pseudo-Wigner-Ville distribution (SPWVD), the spectrogram (SP), the Choi-Williams distribution (CWD), and the modified B-distribution (MBD) distributions. The first three are widely used TFDs. The last one is a recent addition to the quadratic class of TFDs that showed promising results in achieving high TF resolution and significant cross-term reduction [17]. Table 1 shows the TFDs used along with the corresponding kernels [17].

The Wigner-Ville distribution (WVD) with the kernel equal to 1 provides a high-resolution representation of the signal $x(t)$ in time and frequency [17]. The main drawback with the WVD is the presence of cross-terms if the signal is multicomponent such as the HRV. This could be reduced by time and frequency averaging such as in the SPWVD [17]. The SPWVD has separable kernel, where the window $g(t)$ is the smoothing window and the $h(\tau)$ is the analysis window. The $g(t)$ and $h(\tau)$ are chosen to suppress spurious peaks and to obtain a high TF resolution. The suppression of cross-term is better with a longer window. This, however, results in the undesirable smearing of instantaneous characteristics. The commonly used functions for $g(t)$ and $h(\tau)$ are the unit rectangular function and the Gaussian window, respectively [18]. The MBD has a lag independent kernel which means that the filtering is only performed in the time directions [17]. $\beta$ is a real parameter between 0 and 1 that defines the sharpness of the cutoff between cross-terms and autoterms present in the TFD. MBD has been found to be highly suitable for this type of signals, that is, HRV which is multicomponent, and their frequency content varies slowly with time [19]. The CWD has a real parameter $\sigma$ which allows one to select the amount of filtering in the TF domain [17]. 


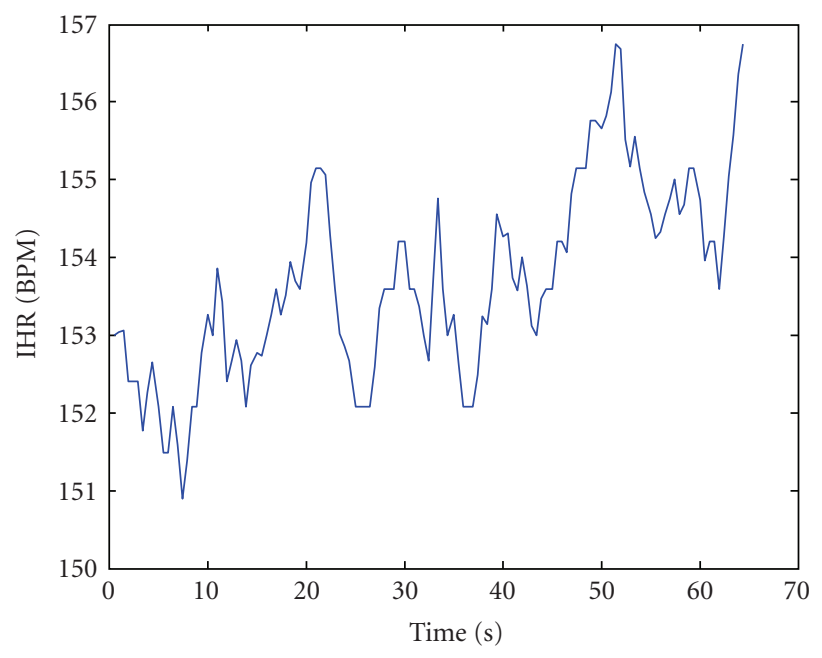

(a)

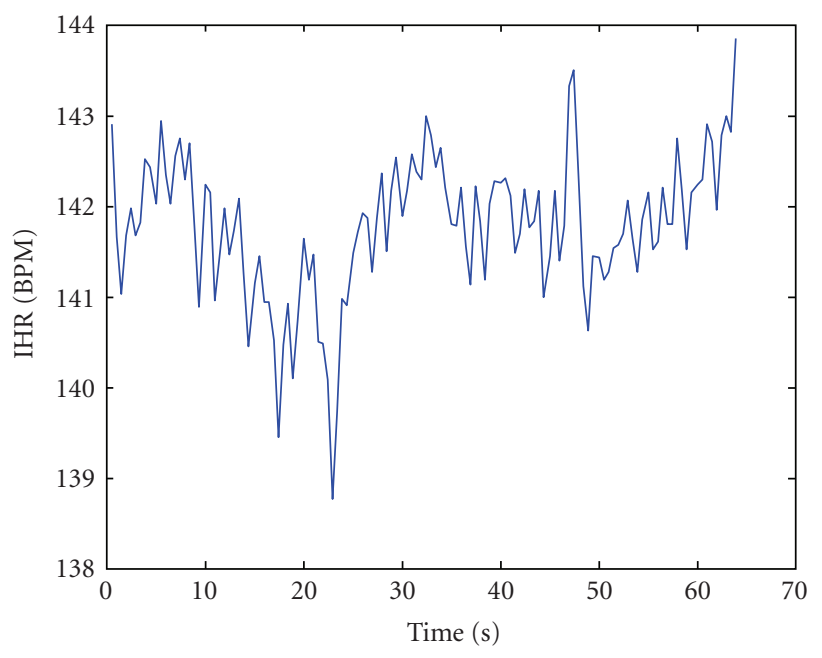

(b)

FIGURE 1: The HRV related to (a) nonseizure EEG and (b) seizure EEG.

The $n$th conditional moment of the TFD at time $t$ is defined as

$$
f_{n}(t)=\frac{1}{P(t)} \int f^{n} \rho_{z}(t, f) d f
$$

where

$$
P(t)=\int \rho_{z}(t, f) d f
$$

The first conditional moment corresponds to the mean or central frequency and the second conditional moment corresponds to the variance. The central/mean frequency $f_{c}(t)$ and variance $\operatorname{var}(t)$ are defined as

$$
\begin{gathered}
f_{c}(t)=\frac{1}{P(t)} \int f \rho_{z}(t, f) d f \\
\operatorname{var}(t)=\frac{1}{P(t)} \int\left(f-f_{c}(t)\right)^{2} \rho_{z}(t, f) d f .
\end{gathered}
$$

\section{METHODS}

The following subsections explain the methods involved in this study.

\subsection{Data acquisition}

The one-channel newborn ECG was recorded simultaneously along with 20 channels of EEG. The EEG was labeled as either seizure or nonseizure by a neurologist from the Royal Children's Hospital, Brisbane, Australia. In the present study, we analyzed 6 seizure events and 4 nonseizure events of 64 seconds each from 5 different newborns. The ECG was sampled at $256 \mathrm{~Hz}$.

\subsection{Preprocessing of ECG for HRV quantification}

The ECG signal is preprocessed to extract the HRV using the following two steps.

\section{QRS detection}

A QRS detection algorithm is used to extract the R points of the ECG. This is the most sensitive parameter in obtaining accurate RR intervals. Conventional time-domain methods, like the ones used in $[8,20]$, are based on differentiation to enhance the peaks in the ECG signal and rule-based thresholding to identify the R points. However, as reported in [21], these methods lead to inaccuracies in the identification and detection of ECG parameters and in certain cases completely miss the QRS waves. In this paper, we used the smoothed nonlinear energy operator (SNEO) to extract the R point which is treated here as a spike in ECG signal. The SNEO has been proposed in $[22,23]$ for the detection of spikes in signals. SNEO is a smoothed version of the nonlinear energy operator (NEO). NEO also is known as the energy-tracking operator. Only three samples are required for energy computation at each time instant. This gives a good time resolution in capturing the energy fluctuations instantaneously.

\section{HRV computation}

The time series of RR interval is called tachogram. Errors in peak detection are corrected based on timing analysis rather than amplitude analysis. Missing beats were estimated and inserted and extra beats were removed based on timing information. The unevenly sampled RR intervals were interpolated using cubic splines. The instantaneous heart rate (IHR) is the inverse of the RR interval and shows the variability of heart rate. Figure 1 shows examples of IHR coinciding with the nonseizure and seizure EEG from the same newborn. An 


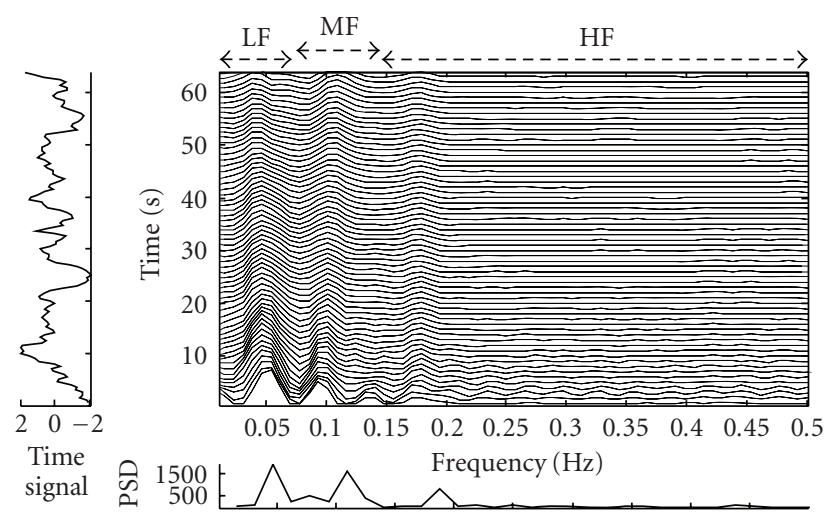

(a) Smoothed pseudo-Wigner-ville

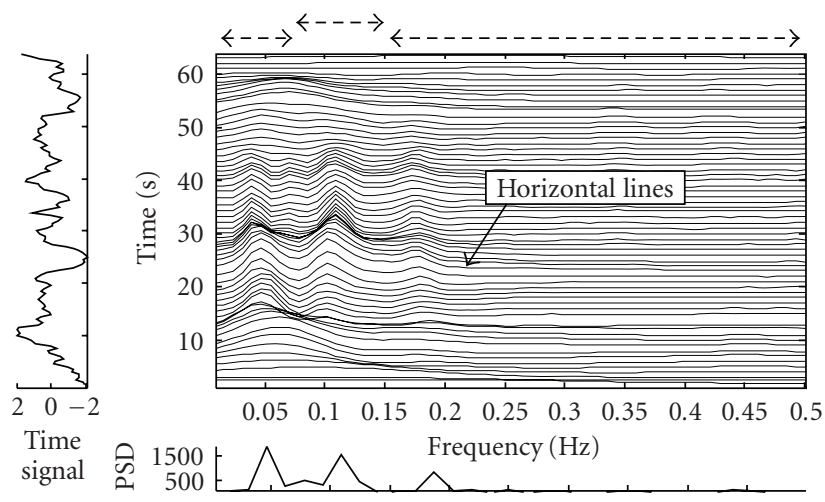

(c) Choi and William

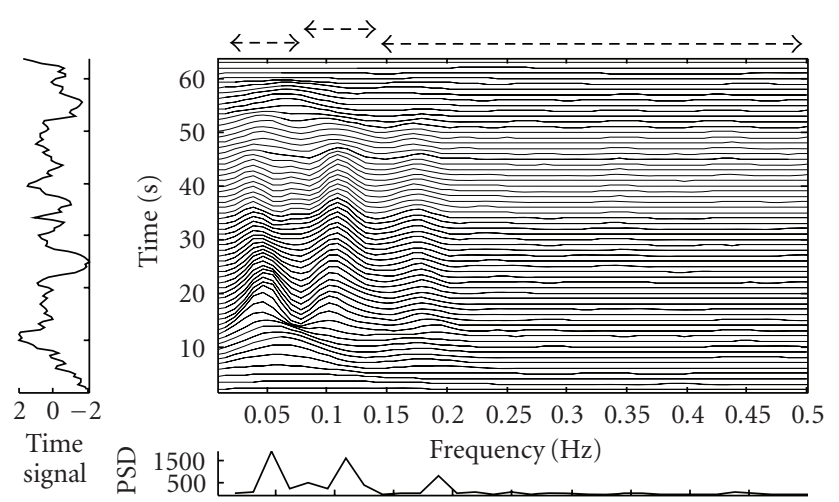

(b) Spectogram
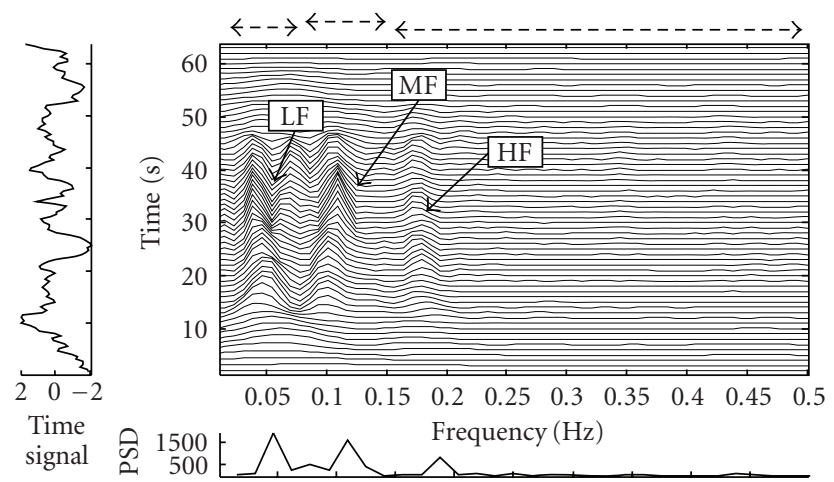

(d) Modified B-distribution

FIgURE 2: TFD for HRV related to nonseizure: (a) SPWVD; (b) SP; (c) CWD and (d) MBD.

antialiasing filter with a cutoff at $1 \mathrm{~Hz}$ was used to filter, and the filtered signals were sampled at a sampling rate of $2 \mathrm{~Hz}$. Finally, the linear trend of the time series was removed. The outcome of the preprocessing stage constitutes the HRV used in the analysis.

\subsection{Selection of the optimal TFD to represent HRV}

The TF analysis was restricted to the SPWVD, the SP, the CWD, and the MBD. Because of the space limitation, we present and discuss the performance analysis using only two signals ( 1 nonseizure and 1 seizure) out of the 10 events studied. These can be considered as representatives of the general characteristics observed. The TFDs of HRV for both the nonseizure and seizure signals in Figure 1 are shown in Figures 2 and 3 , respectively.

All the plots shown were obtained using the same plot routine: the left plot represents time series of HRV and the center figure shows the joint TFD. The sequence of plots labeled with (a), (b), (c), and (d) corresponds to the TFDs of the SPWVD, SP, CWD, and MBD, respectively. For clarity of illustration, the relevant frequency bands are labeled with LF, MF, HF only on Figures 2(d), and 3(d). Because the relative position of those frequencies prevails in all the sequence of figures, the arrows are indicated in Figure 2 only.

The optimal parameters for SPWVD, SP, CW, and MBD are the ones that achieve the best compromise between the TF resolution and the cross-terms suppression. The parameters were selected by comparing the TF plots of the signal visually for different values of parameters. For SPWV, $h(\tau)$ was chosen as a Gaussian window of 121 samples and $g(t)$ as rectangular window of 63 samples. In Figure 2(a), the dominant frequency content can be observed in the LF, MF, and HF. The frequency resolution is fairly satisfactory and its crossterms free. This result is consistent with the findings in [18].

For SP, a Hamming window with length of 111 was used. In Figure 2(b), better defined frequency components can be observed in the MF and HF. However, the SP lacks in time resolution which makes the TF components smeared. The SP smoothes away all interference terms except those occurring when two signal components overlap. As mentioned in Section 1, this smoothing has the side effect of reducing signal components resolution. The SP poorly represents rapidly changing spectral characteristics and cannot optimally resolve closely spaced components. For CWD, the optimal parameter $\sigma$ of its kernel was found to be 0.4 . It can be seen that 

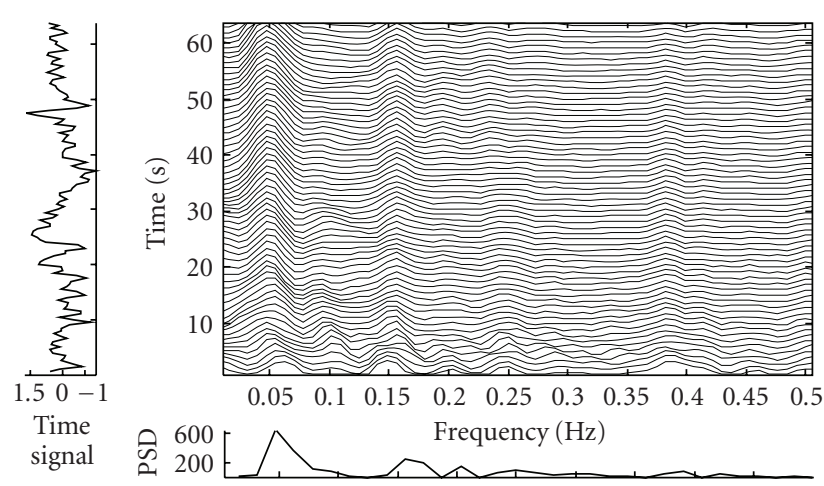

(a) Smoothed pseudo-Wigner-ville

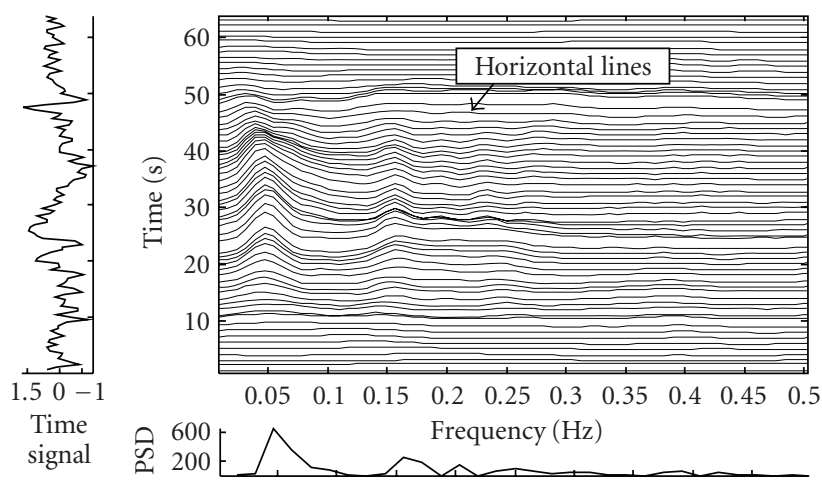

(c) Choi and William

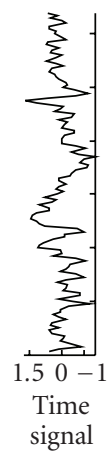

Time

की 600

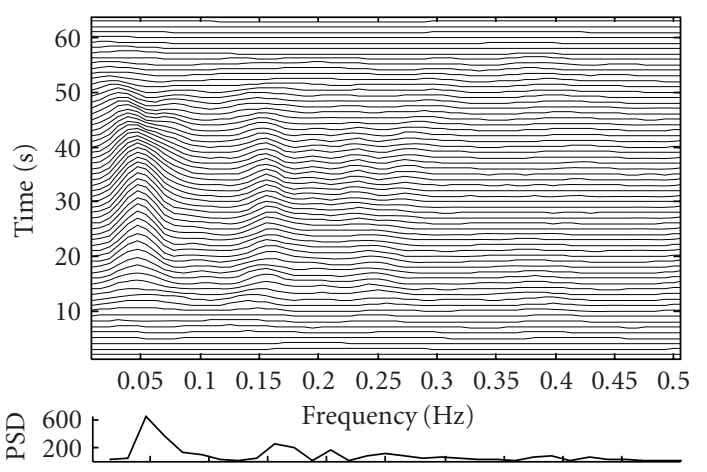

(b) Spectogram
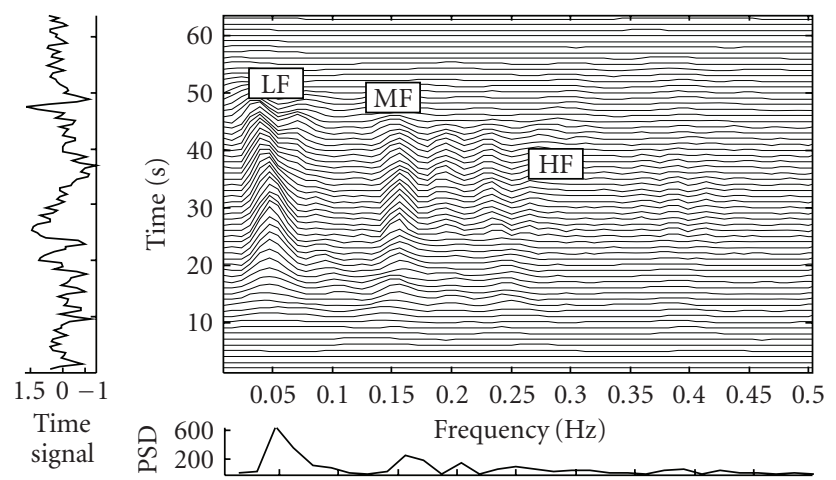

(d) Modified B-distribution

FIgURE 3: TFD for HRV related to seizure: (a) SPWVD; (b) SP; (c) CWD; and (d) MBD.

it is almost cross-terms free but the horizontal lines prevail, which makes the TF components smeared. This is due to the trade-off between suppression of the cross-terms and the resolution of autoterms. This makes the component in LF and MF smeared.

For MBD, the parameter $\beta$ was set to 0.01 . We can see that its cross-terms are free and have better TF resolution compared to SP and CWD. This improvement facilitates the identification/interpretation of the frequency components of the HRV in nonseizure neonatal. The dominant frequency content can be observed in the LF, MF, and HF band. The MBD also gives a good estimation of the instantaneous frequency (IF) law of each component which varies slowly with time. This is consistent with the findings in [19]. The MBD has high TF resolution and is effective in cross-terms reduction.

Results of the TFD analysis of the HRV for seizure baby are presented in Figure 3. Similar patterns are observed regarding the TF resolution and suppression of cross-term interference, as in the case of nonseizure HRV. To better appreciate the performance of the $\mathrm{MBD}$, we compare the frequency resolution using a time slice of TFDs, taken at specific time, $t$. For each TFD for the nonseizure case, a normalized slice at time interval $t=23$ seconds is taken and displayed in Figure 4. This figure shows the normalized slices of TFDs plotted in Figure 2.

From Figure 4(a), the SPWVD shows almost similar performance as the MBD in cross-terms suppression but MBD performs better in preserving the energy concentration for each component and has better TF resolution. The SP too fails to preserve the energy concentration for each component and has poorer TF resolution compared to MBD. Meanwhile, the CWD failed to exhibit a good suppression of any undesirable artifacts for each of the components. Thus, the MBD is found to realize the best compromise for the class of signals considered; it is almost cross-terms free and has high components' resolution in the TF plane. So for this, the MBD will be used in the remaining part of the study.

\subsection{TF feature extraction of HRV}

The parameters derived from the first and second conditional moments of TFD of the HRV signal in each one of the 3 bands will be used as features in discriminating the seizure from the nonseizure. The first conditional moment corresponds to the mean or central frequency $f_{c}(t)$ of the 


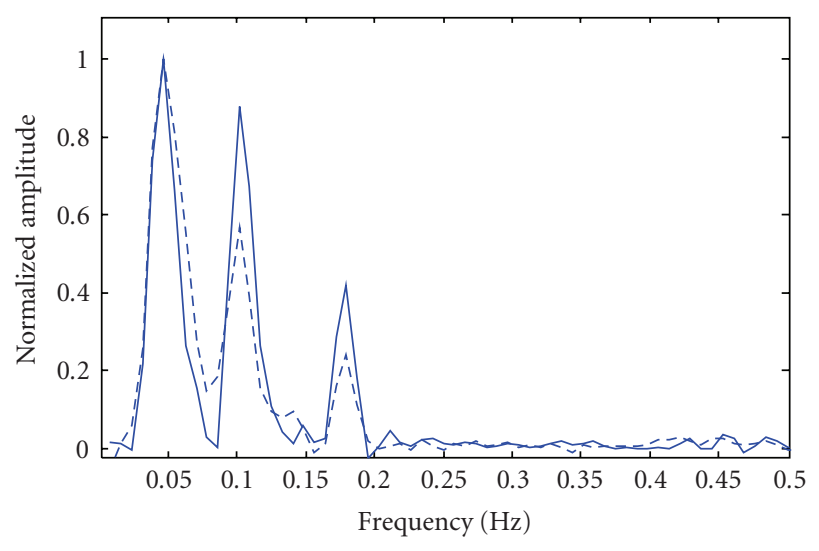

(a)

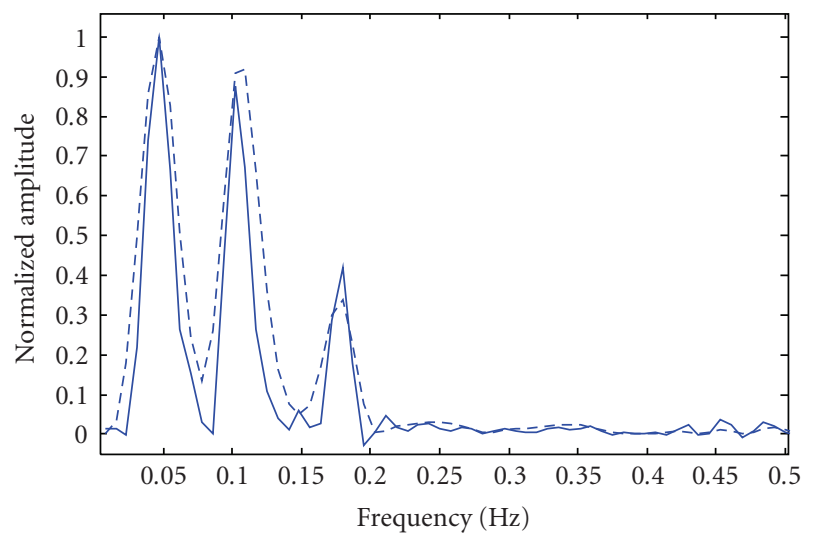

(b)

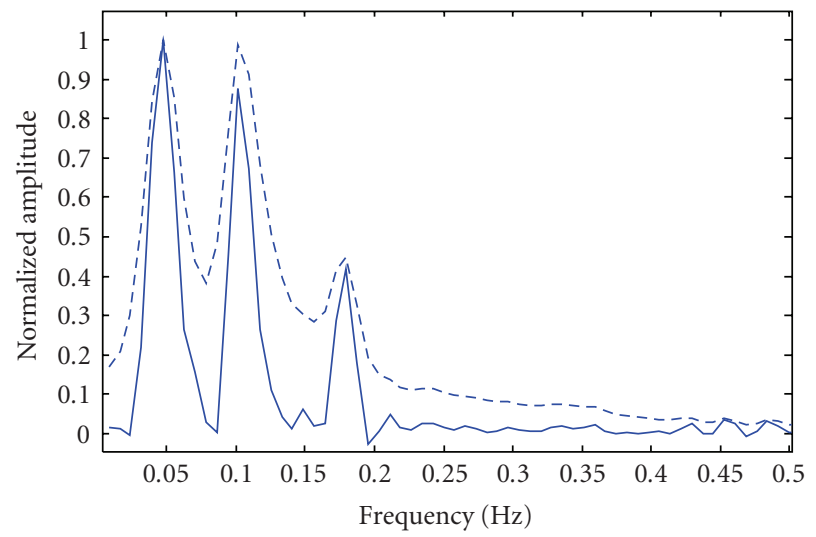

(c)

FIGURE 4: Normalized slices (dashed) of (a) SPWVD; (b) SP; and (c) CWD. All plots are compared against the MBD (solid).

respective spectrum of interest at a particular time and the parameter from second conditional moment corresponds to the variance $\operatorname{var}(t)$. It is worth mentioning that the $f_{c}(t)$ and $\operatorname{var}(t)$ represent, respectively, the instantaneous frequency (IF) and the instantaneous bandwidth (IB) for the case of TFDs whose kernel satisfies the IF property [20]. Unfortu- nately, this is not the case for MBD. Hence, the notions of IF and IB are not used here.

The feature extraction procedure includes the following steps.

(1) Bandpass filtering: FIR bandpass filters are used to isolate the three frequency bands mentioned above; namely LF (0.03-0.07 Hz), MF (0.07-0.15 Hz), and HF $(0.15-0.6 \mathrm{~Hz})$. This results in three filtered signals.

(2) TF mapping: the three filtered signals are mapped using MBD. This step results in three TFDs.

(3) Moment estimation: the $f_{c}(t)$ and the var $(t)$ are computed for each signal. The $f_{c}(t)$ and the $\operatorname{var}(t)$ related to LF, MF, and HF are shown in Figures 5 and 6 respectively.

From these figures, it can be seen that for the case of seizure, the central frequency $f_{c}(t)$ related to LF, MF, and HF occur at frequency higher than the ones appearing in nonseizure. It is the same case for the variance. These facts will be exploited in our seizure detection using HRV.

\section{PERFORMANCE EVALUATION AND DISCUSSION}

Based on the results of the previous section, we will use $f_{c}(t)$ and $\operatorname{var}(t)$ related to the three frequency bands LF, MF, and HF as features to differentiate between seizure and nonseizure. Because not enough data is available at this stage, we opt for the leave-one-out cross-validation method [24]. Given a dataset of size $N$, this method simply consists of splitting the dataset in a set of $N-1$ training data and one test data. So, for 9 events (seizure and nonseizure) at a time, the $f_{c}(t)$ values for seizure were compared with those from nonseizure, and a threshold was chosen that best differentiated the two groups. The threshold is determined using the Gaussian distribution since the values of $f_{c}(t)$ were shown to obey the Gaussian distribution when tested for normality [25]. Figures 7 and 8 show how the threshold is obtained. The one $f_{c}(t)$ which was not included in the training group of 9 was then compared with the obtained threshold and the classification results are noted. The procedure was applied 10 times for both $f_{c}(t)$ and $\operatorname{var}(t)$ related to the three frequency bands. From Figures 7 and 8, for the case shown in Figures 5 and 6 , the optimal threshold was found to be $0.0455 \mathrm{~Hz}$ (for LF) and $0.003 \mathrm{~Hz}^{2}$ (for $\mathrm{HF}$ ), respectively. The threshold selected is different for the different tests (newborn-dependent). The results of the different tests were used to calculate the sensitivity $R_{\mathrm{sn}}$ and specificity $R_{\mathrm{sp}}$.

The sensitivity $R_{\mathrm{sn}}$ and specificity $R_{\mathrm{sp}}$ are defined as

$$
R_{\mathrm{sn}}=\frac{\mathrm{TP}}{\mathrm{TP}+\mathrm{FN}} ; \quad R_{\mathrm{sp}}=\frac{\mathrm{TN}}{\mathrm{TN}+\mathrm{FP}}
$$

where $\mathrm{TP}, \mathrm{TN}, \mathrm{FN}$, and $\mathrm{FP}$, respectively represent the numbers of true positive, true negative, false negative, and false positive. The $R_{\mathrm{sn}}$ is the proportion of seizure events correctly recognized by the test (the seizure detection rate) while $R_{\mathrm{sp}}$ is the proportion of nonseizure events correctly recognized 


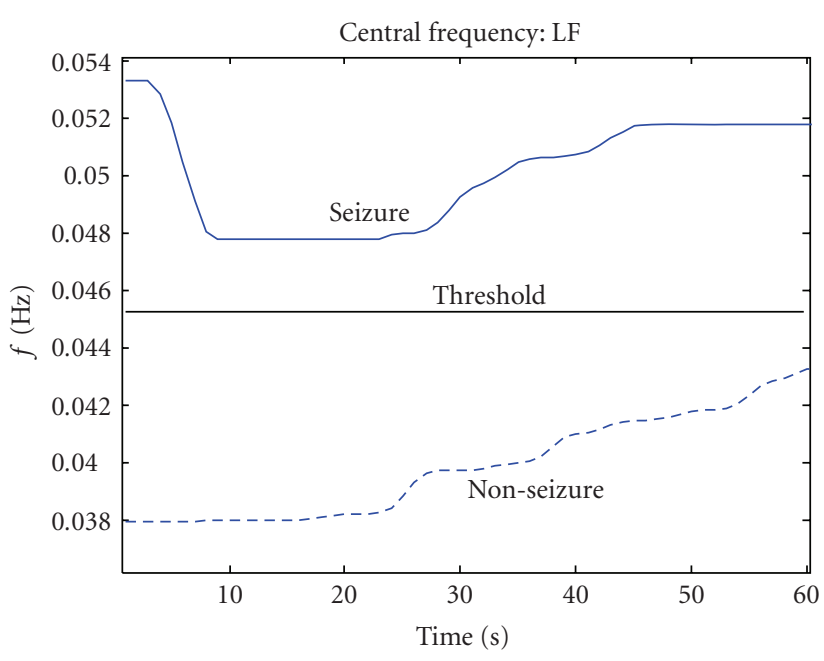

(a)

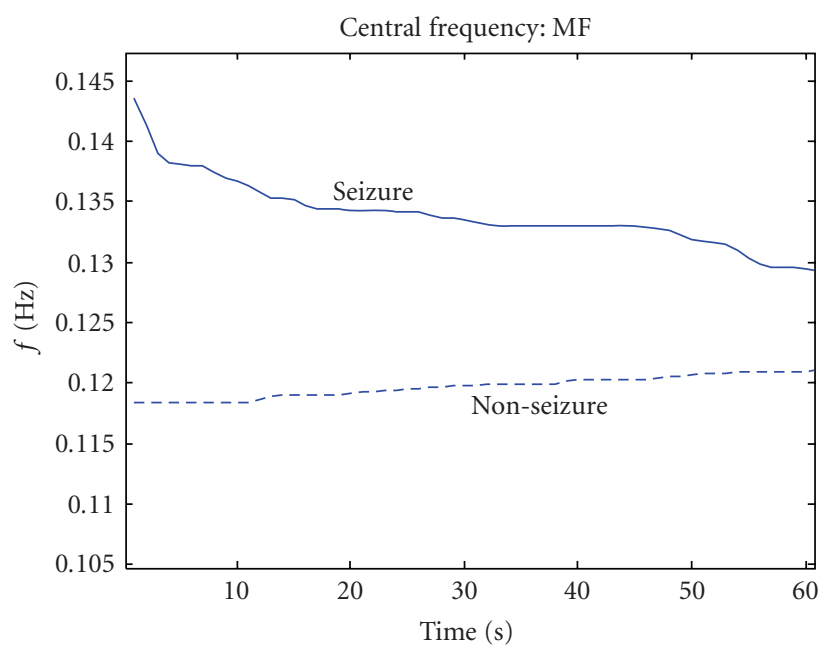

(b)

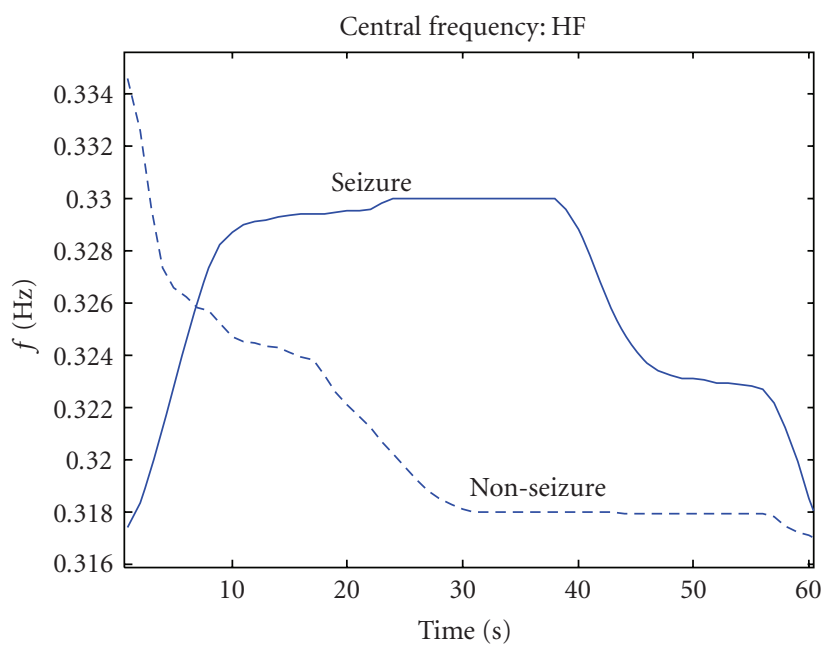

(c)

Figure 5: The central frequency of the LF, MF, and HF of the HRV.

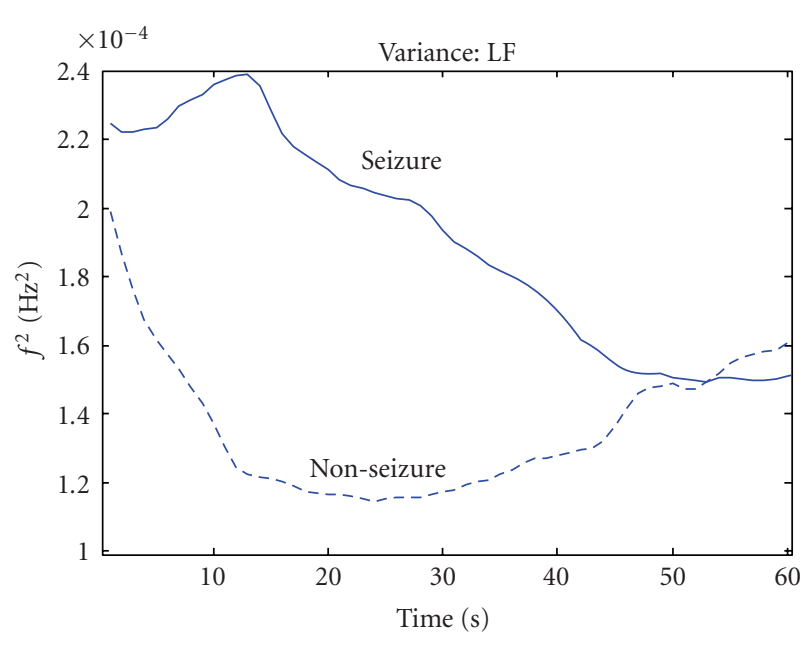

(a)

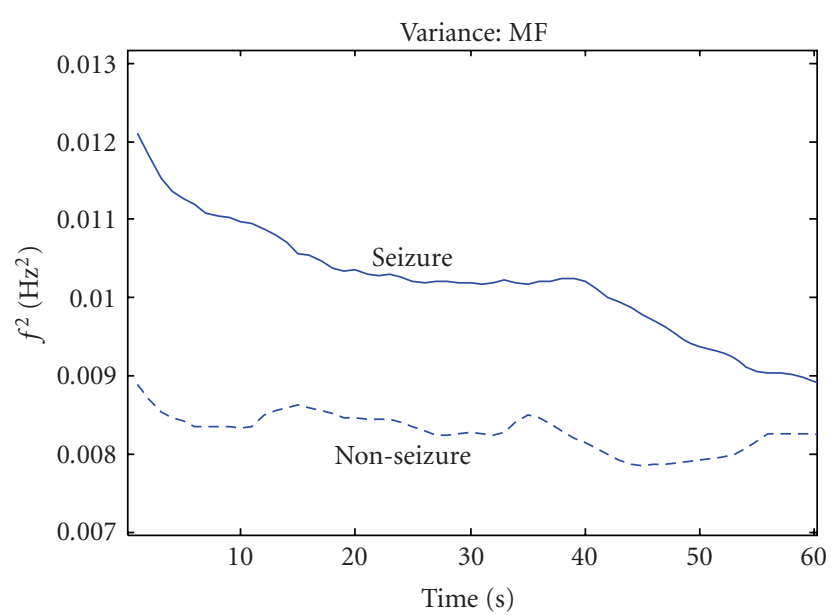

(b)

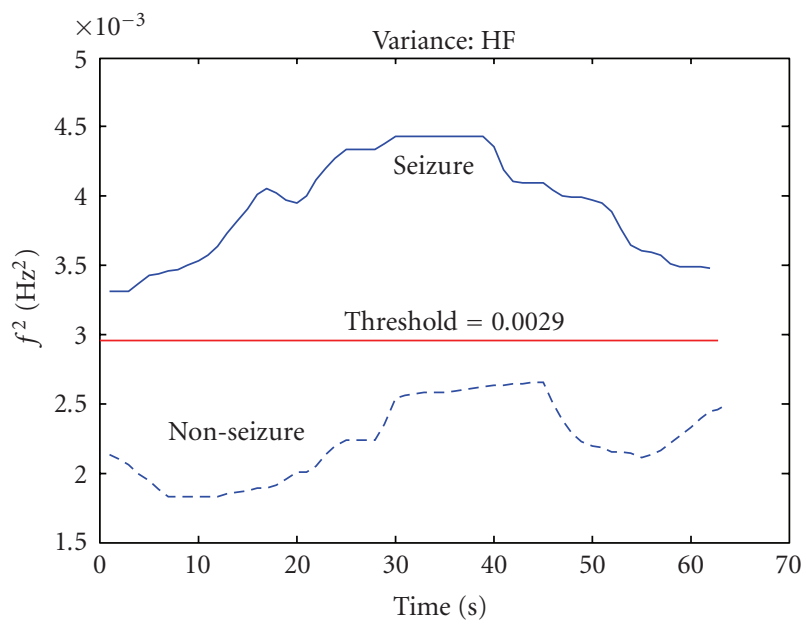

(c)

Figure 6: The variance of the LF, MF, and HF of the HRV. 


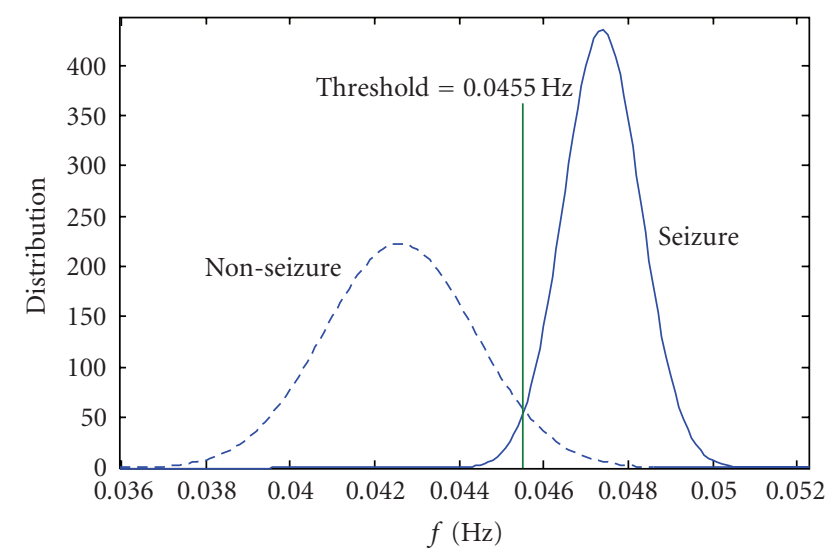

Figure 7: The Gaussian distribution to determine threshold for central/mean frequency in LF.

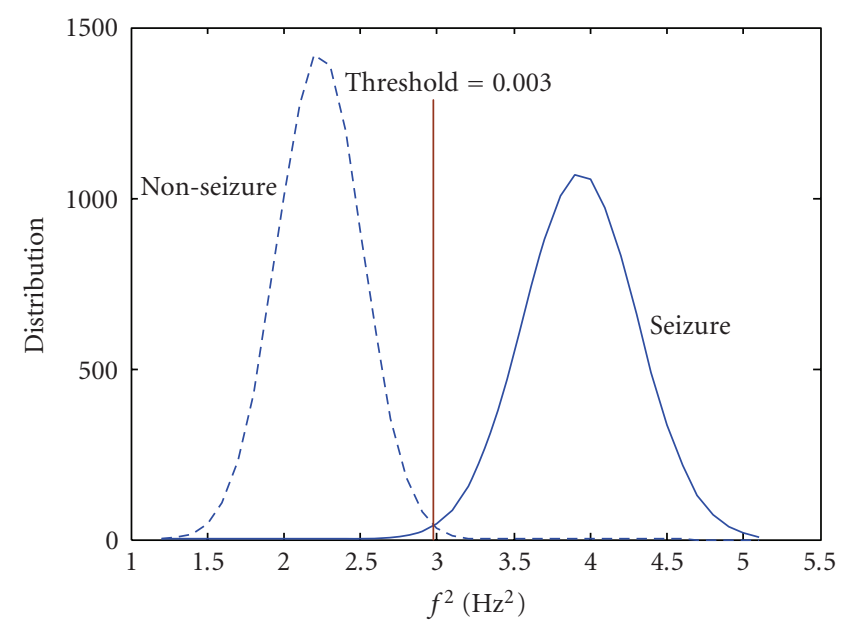

FIgURE 8: The Gaussian distribution to determine threshold for variance in $\mathrm{HF}$.

by the test (the non-seizure detection rate). Table 2 shows the results using $f_{c}(t)$ while Table 3 shows the results using $\operatorname{var}(t)$.

From Table 2, it can be seen that the seizures can best be discriminated from the nonseizure using $f_{c}(t)$ in the LF band $(83.33 \%$ of sensitivity and $100 \%$ of specificity). The optimal averaged threshold was found to be $0.0453 \mathrm{~Hz}$. These results tend to indicate that the newborn seizure manifest itself in the LF component (sympathetic activity) of the HRV the most. The MF component was more affected than HF because it is both parasympathetically and sympathetically mediated. $f_{c}(t)$ from the HF band shows very poor performance. This tends to indicate that the seizures have the least effect in the parasympathetic activity.

For the $\operatorname{var}(t)$, as can be seen in Table 3, the nonseizure can be discriminated clearly from the seizure in the HF band ( $83.33 \%$ of sensitivity and $100 \%$ of specificity). The optimal averaged threshold found was $0.0026 \mathrm{~Hz}^{2}$. These results show
TABLE 2: Results for the central/mean frequency.

\begin{tabular}{l|cr}
\hline Frequency band & $R_{\mathrm{sn}}$ & \multicolumn{1}{c}{$R_{\mathrm{sp}}$} \\
\hline LF & $83.33 \%$ & $100.00 \%$ \\
MF & $83.33 \%$ & $66.67 \%$ \\
HF & $50.00 \%$ & $16.67 \%$ \\
\hline
\end{tabular}

TABLE 3: Results for the variance.

\begin{tabular}{l|cr}
\hline Frequency band & $R_{\mathrm{sn}}$ & \multicolumn{1}{c}{$R_{\mathrm{sp}}$} \\
\hline LF & $66.67 \%$ & $66.67 \%$ \\
MF & $83.33 \%$ & $66.67 \%$ \\
HF & $83.33 \%$ & $100.00 \%$ \\
\hline
\end{tabular}

that $\operatorname{var}(t)$ related to the HF has been affected greatly during seizure compared to those from the LF and MF. The HF band is mediated by the respiration rate. So, these results indicate that the newborn with seizure tends to have higher respiration variation compared to the nonseizure ones. It is worth noting while the $f_{c}(t)$ in the HF is less affected by seizure, the spread of the frequency in this band shows significant difference between them. $\operatorname{var}(t)$ obtained from the LF and MF bands did not show considerable changes. Thus, those features do not seem to be good discriminating features. Based on the results obtained so far, it can be seen that only the two extreme values of both $f_{c}(t)$ and $\operatorname{var}(t)$, namely the maximum and minimum, are needed to distinguish between seizure and nonseizure. This means that the automatic classifier is computationally very efficient.

\section{CONCLUSIONS}

Our aim in this paper was to show that, beside EEG, other physiological signals such as ECG could be used as additional factors in the process of newborn seizure detection. Our long-term goal is to combine features extracted from the different physiological signals to realize accurate and robust automatic seizure detection method. The results so far obtained using HRV show that the first- and second-order TFD moments are potentially good features in the discrimination between seizure and nonseizure. Currently, other timefrequency-based features such as IF are being tested to assess their performance. The identified discriminating features will also be tested using a much larger database once this becomes available later.

\section{ACKNOWLEDGMENTS}

The authors wish to thank Professor Paul Colditz from the Royal Women's Hospital in Brisbane, Australia for providing access to the Perinatal Research Centre; and Dr. Chris Burke and Ms. Jane Richmond from the Royal Children's Hospital in Brisbane, Australia for their assistance for the labeling and interpretation of the EEG data used in this study. 
This study is partly supported under of a project funded by the Australian Research Council's Discovery funding scheme (DP0665697).

\section{REFERENCES}

[1] J. M. Rennie, "Neonatal seizures," European Journal of Pediatrics, vol. 156, no. 2, pp. 83-87, 1997.

[2] A. Liu, J. S. Hahn, G. P. Heldt, and R. W. Coen, "Detection of neonatal seizures through computerized EEG analysis," Electroencephalography and Clinical Neurophysiology, vol. 82, no. 1, pp. 30-37, 1992.

[3] J. Gotman, D. Flanagan, B. Rosenblatt, A. Bye, and E. M. Mizrahi, "Evaluation of an automatic seizure detection method for the newborn EEG," Electroencephalography and Clinical Neurophysiology, vol. 103, no. 3, pp. 363-369, 1997.

[4] B. Boashash and M. Mesbah, "A time-frequency approach for newborn seizure detection," IEEE Engineering in Medicine and Biology Magazine, vol. 20, no. 5, pp. 54-64, 2001.

[5] S. Faul, G. Boylan, S. Connolly, L. Marnane, and G. Lightbody, "An evaluation of automated neonatal seizure detection methods," Clinical Neurophysiology, vol. 116, no. 7, pp. 1533-1541, 2005.

[6] S. R. Quint, J. A. Messenheimer, M. B. Tennison, and H. T. Nagle, "Assessing autonomic activity from the EKG related to seizure onset detection and localization," in Proceedings of the 2nd Annual IEEE Symposium on Computer-Based Medical Systems, pp. 2-9, Minneapolis, Minn, USA, June 1989.

[7] S. J. Tavernor, S. W. Brown, R. M. Tavernor, and C. Gifford, "Electrocardiograph QT lengthening associated with epileptiform EEG discharges - a role in sudden unexplained death in epilepsy?" Seizure, vol. 5, no. 1, pp. 79-83, 1996.

[8] F. Leutmezer, C. Schernthaner, S. Lurger, K. Pötzelberger, and C. Baumgartner, "Electrocardiographic changes at the onset of epileptic seizures," Epilepsia, vol. 44, no. 3, pp. 348-354, 2003.

[9] M. Zijlmans, D. Flanagan, and J. Gotman, "Heart rate changes and ECG abnormalities during epileptic seizures: prevalence and definition of an objective clinical sign," Epilepsia, vol. 43, no. 8, pp. 847-854, 2002.

[10] P. Tinuper, F. Bisulli, A. Cerullo, et al., "Ictal bradycardia in partial epileptic seizures: autonomic investigation in three cases and literature review," Brain, vol. 124, no. 12, pp. 23612371, 2001.

[11] R. N. Goldberg, S. L. Goldman, R. E. Ramsay, and R. Feller, "Detection of seizure activity in the paralyzed neonate using continuous monitoring," Pediatrics, vol. 69, no. 5, pp. 583$586,1982$.

[12] M. E. O'Regan and J. K. Brown, "Abnormalities in cardiac and respiratory function observed during seizures in childhood," Developmental Medicine and Child Neurology, vol. 47, no. 1, pp. 4-9, 2005.

[13] M. V. Kamath, T. Bentley, R. Spaziani, et al., "Time-frequency analysis of heart rate variability signals in patients with autonomic dysfunction," in Proceedings of the IEEE-SP International Symposium on Time-Frequency and Time-Scale Analysis, pp. 373-376, Paris, France, June 1996.

[14] J. P. Finley and S. T. Nugent, "Heart rate variability in infants, children and young adults," Journal of the Autonomic Nervous System, vol. 51, no. 2, pp. 103-108, 1995.

[15] R. M. S. S. Abeysekera, Time-frequency domain features of electrocardiographic signals: an interpretation and their application in computer aided diagnosis, Ph.D. thesis, University of Queensland, Brisbane, Australia, 1989.
[16] B. Tacer and P. J. Loughlin, "Non-stationary signal classification using the joint moments of time-frequency distributions," Pattern Recognition, vol. 31, no. 11, pp. 1635-1641, 1998.

[17] B. Boashash, Time Frequency Signal Analysis and Processing: A Comprehensive Reference, Elsevier, Oxford, UK, 2003.

[18] P. Novak and V. Novak, "Time/frequency mapping of the heart rate, blood pressure and respiratory signals," Medical and Biological Engineering and Computing, vol. 31, no. 2, pp. 103-110, 1993.

[19] L. Rankine, M. Mesbah, and B. Boashash, "Resolution analysis of the T-class time-frequency distributions," in Proceedings of the International Symposium on Signal Processing and Its Applications (ISSPA '07), Sharjah, United Arab Emirates, February 2007.

[20] B. Boashash, "Time-Frequency Signal Analysis," in Advances in Spectrum Estimation and Array Processing, S. Haykin, Ed., chapter 9, pp. 418-517, Prentice-Hall, Englewood Cliffs, NJ, USA, 1990.

[21] T. Srikanth, S. A. Napper, and H. Gu, "Bottom-up approach to uniform feature extraction in time and frequency domains for single lead ECG signal," International Journal of BioElectromagnetism, vol. 4, no. 1, 2002.

[22] S. Mukhopadhyay and G. C. Ray, "A new interpretation of nonlinear energy operator and its efficacy in spike detection," IEEE Transactions on Biomedical Engineering, vol. 45, no. 2, pp. 180-187, 1998.

[23] H. Hassanpour and M. Mesbah, "Neonatal EEG seizure detection using spike signatures in the time-frequency domain," in Proceedings of the 7th International Symposium on Signal Processing and Its Applications (ISSPA '03), vol. 2, pp. 41-44, Paris, France, July 2003.

[24] S. Theodoridis and K. Koutroumbas, Pattern Recognition, Academic Press, San Diego, Calif, USA, 2006.

[25] H. L. Macgillivray, Data Analysis: Introductory Methods in Context, Queensland University of Technology, Brisbane, Australia, 2004.

M. B. Malarvili received both the B.Eng and M.Eng degrees in electrical engineering from Universiti Teknologi of Malaysia at Skudai, Johor, Malaysia, in 2001 and 2004, respectively. She is currently doing her Ph.D. degree in biomedical signal processing at the Perinatal Research Centre (PRC), The University of Queensland in Brisbane, Australia. Her research interests include biomedical signal processing, pattern recognition, and time-frequency signal analysis.

Mostefa Mesbah received his M.S. and Ph.D. degrees in electrical engineering from University of Colorado at Boulder, Colo, USA, in the area of automatic control systems. He is currently a Research Fellow at the Perinatal Research Centre (PRC), The University of Queensland in Brisbane, Australia, leading biomedical engineering projects that deal with the automatic detection and classification of newborn EEG

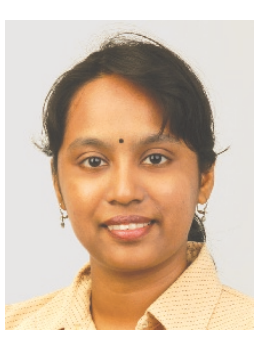
seizures. His research interests include biomedical signal processing, time-frequency signal processing, signal detection and classification, 3D shape reconstruction from image sequences, and intelligent control systems. 
Boualem Boashash obtained a Diplome d'Ingenieur-Physique-Electronique from Institut de Chimie et de Physique Industrielles de Lyon (ICPI), University of Lyon, France, in 1978, the M.S. and Doctorate (Docteur-Ingenieur) degrees from the Institute National Polytechnique de Grenoble, France, in 1979 and 1982, respectively. In 1979, he joined Elf-Aquitaine Geophysical Research Centre, Pau, France. In May

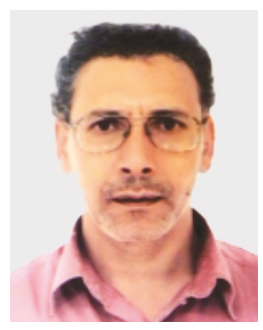
1982, he joined the Institut National des Sciences Appliquees de Lyon, France. In 1984, he joined the Electrical Engineering Department, University of Queensland, Australia, as a Lecturer. In 1990, he joined Graduate School of Science and Technology, Bond University, as a Professor of electronics. In 1991, he joined Queensland University of Technology as the Foundation Professor of signal processing and Director of the Signal Processing Research Centre. In 2006, he joined the Perinatal Research Centre (PRC), The University of Queensland in Brisbane, Australia, as a Research Fellow and also as the Dean of the College of Engineering in University of Sharjah, UAE. B. Boashash is the Editor of three books and has written over four hundred technical publications. His research interests include time-frequency signal analysis, spectral estimation, signal detection and classification, and higher-order spectra. Professor Boashash is a Fellow of Engineers of Australia, Fellow of IREE, and Fellow of IEEE. 